\section{Pflichtlektüre für Allergologen}

A us dem Taschenbuch der Erstauflage ist inzwischen ein umfangreiches Werk von 392 Seiten geworden handlich zwar noch, aber deutlich gewichtiger als seine Vorgänger. Wenn als Erscheinungsjahr 2004 genannt ist, dann spiegelt dies die Aktualität des Werkes wider. Dem Autor und seinen Mitarbeitern sowie dem Verlag ist zu der inhaltlich und optisch gelungenen Neuauflage zu gratulieren!

Kein wichtiges Gebiet der Allergologie ist ausgespart. Genetik, pathophysiologische Grundlagen, Diagnostik und Therapie der klinischen Krankheitsbilder sind nach neuesten Erkenntnissen dargestellt. Immer aber fließt die große Erfahrung des Autors mit ein und es wird auch auf ältere Quellen zurückgegriffen. Naturgemäß sind die Abschnitte über dermatologische Krankheitsbilder und Forschungsschwerpunkte von besonderer Aktualität nicht zuletzt finden sich auch oft ausgesparte Themen wie das Öko-Syndrom beziehungsweise die multiple Chemikalien-Sensitivitität oder Psyche und Allergie. Aber auch zu Asthma und exo- gen-allergischer Alveolitis sowie zu Nahrungsmittelund Insektengiftallergie sind umfangreiche Abschnitte in dem Kompendium enthalten.

Über die auf konkrete Krankheitsbilder bezogenen Darstellungen hinaus sind übergreifende Kapitel der Allergiediagnostik, -prävention und -therapie gewidmet und auch unkonventionelle Verfahren werden kommentiert. Ein Ausblick verweist auf die Zukunft der Allergologie, ein Anhang liefert wichtige Anschriften und Literaturhinweise.

Die Auswahl der Tabellen und Abbildungen unterstützt die Texte sehr wirkungsvoll - man nimmt das Werk gern in die Hand. Der Spezialist wird hier und da eventuell etwas vermissen oder sich andere Akzente wünschen für die interessierten Leser aller allergologischen Disziplinen und insbesonde-
Ring J.

Angewandte

Allergologie.

München:

Urban \& Vogel,

2004.

$69,95 \mathrm{~g}$.

ISBN 3-89935-

128-2
Angewandte Allergologie

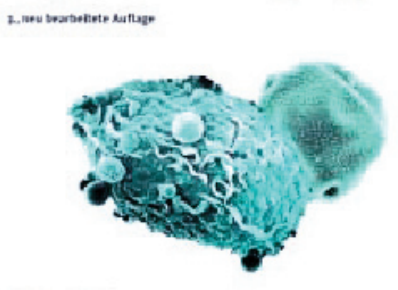

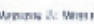

\title{
Kompetente Kontaktekzem-Kollektion
}

U nter Federführung der beiden Herausgeber Prof. Dr. Thomas Fuchs, Göttingen, und Prof. Dr. Werner Aberer, Graz, ist ein einmaliges deutschsprachiges Buch zum Thema Kontaktekzem entstanden. In 47 Einzelkapiteln wird in diesem „Multiautorenwerk" vielfältigen Aspekten Rechnung getragen: aktuelles Grundlagenwissen, klinische Erscheinungsformen, diagnostische Methoden, Informationen zu Kontaktallergenen der Epikutanteststandardreihe und Einfluss von Geschlecht und Lebensphase.

Es kommen jeweils ausgewiesene Experten zu Wort und liefern in ihren unterschiedlich gegliederten Kapiteln aktuelle Informationen einschließlich relevanter Literaturhinweise. Zwangsläufig treten dabei gelegentlich Wie- derholungen und Überschneidungen auf, was dieses wertvolle Nachschlagewerk aber nicht beeinträchtigt. Der Leser findet nämlich ausführliche Informationen und Anregungen, die mit dem Konzept der Loseblattsammlung auch einer Aktualisierung unterliegen sollen.

Insgesamt haben die beiden Herausgeber den englischsprachigen Standardwerken ein deutschsprachiges informatives Buch zur Seite gestellt, das einen klar konzipierten Zugang zu ausführlicher Information über wesentliche Aspekte des Kontakt- re den allergologisch Interessierten mit Fragen aller Art liefert die „Angewandte Allergologie“ eine Fülle an Informationen, auf die Verlass ist.

Es gibt derzeit kein anderes, ähnlich empfehlenswertes, kurzgefasstes Buch zum Thema. Es fällt nicht schwer, dem Werk seinen Weg zur Pflichtlektüre vorauszusagen.

Prof. Dr. Gerhard SchultzeWerninghaus, Bochum ekzems ermöglicht. So wird dieses Buch sowohl als Nachschlagewerk wie auch als übersichtlicher Ratgeber viele Nutzer finden.

Priv.-Doz. Dr. Peter Thomas, München

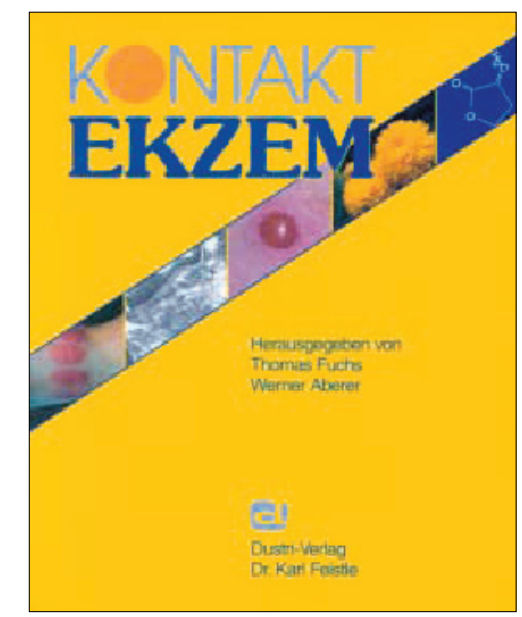

Fuchs T., Aberer W. (Hrsg.). Kontaktekzem. Deisenhofen: Dustri-Verlag, 2002. $137,95 \mathrm{~g}$. ISBN 3-87185 292-9 